\title{
Waiting time distributions in a two-queue model with mixed exhaustive and gated-type $K$-limited services
}

Toshihisa OZAWA

NTT Multimedia Networks Laboratories

3-9-11 Midori-cho, Musashino-shi, Tokyo 180, Japan

E-mail: ozawa@hashi.tnl.ntt.co.jp

\begin{abstract}
We deal with a two-queue model with mixed exhaustive and gated-type $K$ limited services. This model can achieve various degrees of priority in each queue by varying the value of $K$. Analyzing the model by using the delay cycle technique, we obtain the Laplace-Stieltjes transforms of the waiting time distributions for both cases with zero and non-zero walking times.
\end{abstract}

Keywords

Multiqueue model, exhaustive service, $K$-limited service, waiting time

\section{INTRODUCTION}

Several typical queueing models are used in developing a range of service grades. One is a non-preemptive priority model (Jaiswal 1968), which is also 
called a head-of-line priority model. But, this model has two drawbacks: First, it has no means of controling the degree of the priority in each customer class, such as the ratio of the mean waiting times. Therefore, when the offered load of priority customers is high, the waiting times for ordinary customers may become very long and unacceptable, while those of priority customers are unnecessarily short. Second, the switchover time, the time for the server to change from the services of one class to another, is not considered in this model. Hence, in modeling an actual system, if we decide to use a non-preemptive priority model, we always have to assume the switchover time to be zero.

In this paper we deal with a case of two customer classes, and consider an extended version of such a non-preemptive priority model. One way to remove the drawbacks mentioned above is to represent the original priority model as a polling model. A polling model is a system that has multiple queues accessed in cyclic order by a single server (Boxma et al. 1987, Takagi 1986, Takagi 1988). In this model, each queue corresponds to a customer class, and a switchover time exists for the server to move from one queue to another. This switchover time is also called a walking time. We apply a different service discipline to each queue in order to control the degree of the priority in each customer class. Service disciplines can be classified by the number of customers in a queue served during one visit of the server. The following service disciplines are typical:

(i) Exhaustive service. The server serves customers in a queue until the queue becomes empty.

(ii) Gated service. The server continuously serves only those customers in a queue who have arrived before the server's visit.

(iii) $K$-limited service (exhaustive-type). The server serves customers in a queue until either $K$ customers have been served or the queue becomes empty. When the parameter $K$ is large enough, this discipline functions nearly like an exhaustive service.

(iv) $K$-limited service (gated-type). The server serves only $K$ customers in a queue who have arrived before the server's visit. When the parameter $K$ is large enough, this discipline functions nearly like a gated service.

In our model, which is a single-server model with two queues, one queue is served according to an exhaustive service and the other is served according to a gated-type $K$-limited service. Of course, walking time may be non-zero. In a non-preemptive priority model with two classes, the queue of priority customers is served according to an exhaustive service, that of ordinary customers is served according to a 1-limited service, and walking time is assumed to be zero. Thus, our model is an extended version of such a non-preemptive priority model, and it can control the degree of the priority by varying the value of $K$. In our previous paper (Ozawa 1990), the mean waiting times of the model with zero walking times were derived. Here we extend the previous 
paper by 1) deriving the Laplace-Stieltjes transforms (LSTs) of the waiting time distributions for the same model as the previous one, in addition to the mean waiting times, and 2) deriving the LSTs of the waiting time distributions and the mean waiting times for the new model with non-zero walking times.

Models with two queues, like the ones considered here, have been investigated in many papers (Avi-Itzhak et al. 1965, Boxma 1984, Cohen et al. 1981, Eisenberg 1971, Eisenberg 1979, Jaiswal 1968, Lee 1996, Ozawa 1988, Ozawa 1990, Skinner 1967, Takács 1968). However, only a few papers have dealt with $K$-limited service. Lee (1996) analyzed a two-queue model with mixed exhaustive and exhaustive-type $K$-limited services for a case with zero walking times, and obtained the generating function of the joint distribution for the numbers of customers in the queues. As mentioned above, two-queue models are also polling models. Polling models with queues served according to $K$-limited services were analyzed by Everitt (1986), Everitt (1989), and Fuhrmann et al. (1988). Everitt (1986) and Everitt (1989) derived a pseudoconservation law, which is a formula for a certain weighted sum of the mean waiting times at each queue, and approximated the mean waiting times by using this law. Fuhrmann et al. (1988) derived an upper bound of a certain weighted sum of the mean waiting times at each queue, and approximated the mean waiting times by using this bound. Polling models with mixed service disciplines were investigated by Groenendijk (1988), Takagi (1989), and Watson (1984), but these studies did not include models with queues served according to $K$-limited services.

Our model originally arose from a common channel signal transfer system (Ozawa 1990). This system has two priority classes for signals: One is for signals that contain call control messages, and the other is for signals that contain data. If the clocked interruption mechanism of the system can be ignored on its performance evaluation, the signal transfer mechanism is represented as a two-queue model with mixed exhaustive and gated-type $K$-limited services. However, the application of our results should not be restricted to this system; the results are possibly used for designing other information communication systems such as ATM switching systems (Lee 1996), routers of IP packets, and information server systems, in which there exist various kinds of inputs (e.g. calls, packets, and tasks) and each kind of input requires its own service grade.

The rest of the paper is constructed as follows. In section 2 we describe our two-queue model in detail. In section 3 we analyze the model for a case with zero walking times and derive the LSTs of the waiting time distributions. In section 4 we analyze that model for a case with non-zero walking times and derive the LSTs of the waiting time distributions. 


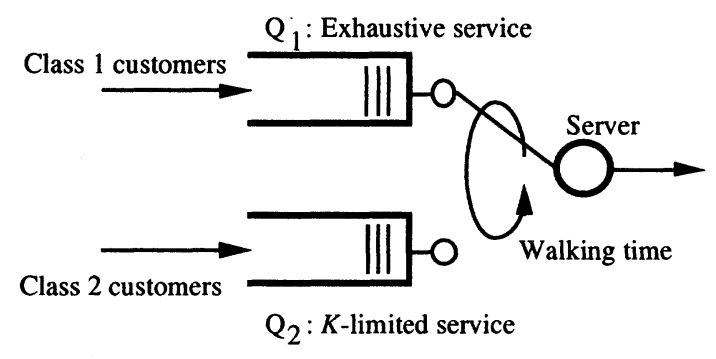

Figure 1 A two-queue model.

\section{MODEL DESCRIPTION}

The model under consideration consists of two queues $\mathrm{Q}_{1}$ and $\mathrm{Q}_{2}$, each with infinite capacity (see Figure 1). Customers arrive at the queues according to independent Poisson processes with intensities $\lambda_{1}$ and $\lambda_{2}$, and the total arrival rate is given by $\lambda=\lambda_{1}+\lambda_{2}$. Customers arriving at $\mathrm{Q}_{i}$ are called Class $i$ customers.

In this model, a single server visits the queues alternately. The service discipline at $\mathrm{Q}_{1}$ is exhaustive and that at $\mathrm{Q}_{2}$ is gated-type $K$-limited. The service times of Class $i$ customers are i.i.d. stochastic variables; their distribution $H_{i}(t)$ has the first moment $h_{i}$ and the second moment $h_{i}^{(2)}$, and its LST is denoted by $H_{i}^{*}(s)$. The walking times from $\mathrm{Q}_{1}$ to $\mathrm{Q}_{2}$ are i.i.d. stochastic variables, and those from $Q_{2}$ to $Q_{1}$ are also i.i.d. stochastic variables; and they are mutually independent. Let $U_{1}(t)$ denote the walking time distribution from $\mathrm{Q}_{1}$ to $\mathrm{Q}_{2}$ and $U_{2}(t)$ denote that from $\mathrm{Q}_{2}$ to $\mathrm{Q}_{1}$. For each $i=1,2$, the LST, the mean, and the second moment of $U_{i}(t)$ are, respectively, $U_{i}^{*}(s), u_{i}$, and $u_{i}^{(2)}$. We assume that the arrival and service processes are mutually independent. The offered load at $\mathrm{Q}_{i}, \rho_{i}$, is defined as $\rho_{i}=\lambda_{i} h_{i}$ for $i=1,2$, and the total offered load, $\rho$, is defined as $\rho=\rho_{1}+\rho_{2}$. We also define the following notations:

$$
u \equiv u_{1}+u_{2}, \quad u^{(2)} \equiv u_{1}^{(2)}+2 u_{1} u_{2}+u_{2}^{(2)}, \quad \omega_{0} \equiv \frac{\lambda_{1} h_{1}^{(2)}+\lambda_{2} h_{2}^{(2)}}{2}
$$

\section{A ZERO WALKING TIME MODEL}

We consider a two-queue model in which the server visits the two queues alternately with zero walking times. Whenever the server finds that one queue is empty, it moves immediately to the other, and if both queues are empty it becomes idle until the next arrival. In this model, the number of customers in $\mathrm{Q}_{1}$ has a steady-state probability if and only if $\rho_{1}<1$; and the number of customers in $\mathrm{Q}_{2}$ has a steady-state probability if and only if $\rho<1$. Hence, 


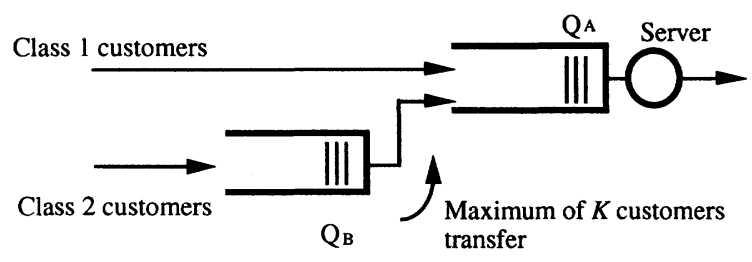

Figure 2 A modified model.

if $\rho_{1}<1$ and $\rho \geq 1$, then $\mathrm{Q}_{1}$ is in steady state and $\mathrm{Q}_{2}$ is saturated. In this case, the waiting time distribution for Class 1 customers can be derived by analyzing an $M / G / 1$ vacation model; its arrival and service processes are the same as those of the original model and each vacation time is the sum of service times of $K$ Class 2 customers. If $\rho<1$, where $\rho_{1}$ is also less than one, then the numbers of customers in $\mathrm{Q}_{1}$ and $\mathrm{Q}_{2}$ both have steady-state probabilities. Hence we assume $\rho<1$ in this section.

Considering the time points when the server departs from $Q_{1}$, when it becomes idle, and when it becomes busy, the number of customers in $\mathrm{Q}_{2}$ forms a one-dimensional piecewise Markov process (Ozawa 1990). The LSTs of the waiting time distributions are obtained by using the steady-state distribution of the embedded Markov chain of the piecewise Markov process.

\subsection{Piecewise Markov process representation}

In this subsection we briefly summarize some results from Ozawa (1990) that are related to our analysis.

\section{(a) A modified model}

To analyze the model, called the original model, a modified model is considered (see Figure 2). The modified model consists of two queues, $\mathrm{Q}_{A}$ and $\mathrm{Q}_{B}$, each with infinite capacity. Class 1 customers arrive directly at $\mathrm{Q}_{A}$. Class 2 customers arrive at $\mathrm{Q}_{B}$ and transfer to $\mathrm{Q}_{A}$ according to the following rules:

- Immediately after all customers in $\mathrm{Q}_{A}$ have been served, a maximum of $K$ Class 2 customers in $\mathrm{Q}_{B}$ transfer to $\mathrm{Q}_{A}$.

- When no customers exist in the system, an arriving Class 2 customer is immediately transferred to $\mathrm{Q}_{A}$.

Zero transfer time is assumed. The service disciplines at both $\mathrm{Q}_{A}$ and $\mathrm{Q}_{B}$ are first-in-first-out (FIFO). Other aspects of the modified model are the same as those of the original model. In this modified model, the movement of the server is replaced by the transfers of Class 2 customers. 
(b) Piecewise Markov process representation

Let $N_{B}(t)$ denote the number of customers in $\mathrm{Q}_{B}$ at time $t$, and $S(t)$ denote the state of the server at time $t$. The state space of $N_{B}(t)$ is a set of nonnegative integers and that of $S(t)$ is $\{I, B\}$, where " $I$ " means that the server is idle and " $B$ " means that it is busy. A joint process $Y(t)$ is defined as follows:

$Y(t) \equiv\left(N_{B}(t), S(t)\right)$.

The state space of $Y(t)$ is $\Omega=\{0 I, 0 B, 1,2, \ldots, j, \ldots\}$, where " $0 I$ " means the state $(0, I)$, " $0 B$ " means the state $(0, B)$, and " $j$ " means the state $(j, B)$, for $j=1,2, \ldots$. Let random variables $t_{0}=0<t_{1}<t_{2}<\ldots$ be the time points when the following state transitions occur:

- $0 B \rightarrow 0 I$ : The system becomes empty.

- $0 I \rightarrow 0 B$ : A customer arrives when the system is empty.

- $j \rightarrow 0 B(1 \leq j \leq K), j \rightarrow j-K(j>K)$ : Class 2 customers transfer to $\mathrm{Q}_{A}$.

An embedded Markov chain for the process $\{Y(t), t \geq 0\}$ is given by

$Y_{n} \equiv Y\left(t_{n}-\right), \quad n \geq 0$

This chain $\left\{Y_{n}, n \geq 0\right\}$ is formed by the states just prior to the time points when the state transitions occur, not by the states just after those time points, in order to make the chain Markovian. From Ozawa (1990), the process $\{Y(t), t \geq 0\}$ is represented as a piecewise Markov process and the points $t_{n}, n \geq 0$ are its regeneration points. A piecewise Markov process is a discretestate, continuous-parameter stochastic process that is Markovian within contiguous time segments (Kuczura 1973).

Here we introduce some notations for the analysis. Let $G_{y}(t) \equiv \operatorname{Pr}\left(t_{n}-\right.$ $\left.t_{n-1} \leq t \mid Y_{n-1}=y\right), y \in \Omega$ be a conditional distribution of a regeneration interval. Note that the subscript $y$ of $G_{y}(t)$ denotes the state just prior to the current state. $G_{y}(t)$ is given by

$$
\begin{aligned}
G_{0 B}^{*}(s) & =\frac{\lambda}{\lambda+s}, \\
G_{0 I}^{*}(s) & =\frac{\lambda_{1}}{\lambda} G^{*}(s)+\frac{\lambda_{2}}{\lambda} H_{2}^{*}\left(s+\lambda_{1}-\lambda_{1} G^{*}(s)\right), \\
G_{j}^{*}(s) & = \begin{cases}\left\{H_{2}^{*}\left(s+\lambda_{1}-\lambda_{1} G^{*}(s)\right)\right\}^{j}, & K>j \geq 1, \\
\left\{H_{2}^{*}\left(s+\lambda_{1}-\lambda_{1} G^{*}(s)\right)\right\}^{K}, & j \geq K,\end{cases}
\end{aligned}
$$


where $G^{*}(s)$ is the LST of the busy period distribution for Class 1 customers, and it is given by the solution of the following functional equation:

$G^{*}(s)=H_{1}^{*}\left(s+\lambda_{1}-\lambda_{1} G^{*}(s)\right)$

(c) Embedded Markov chain $\left\{Y_{n}, n \geq 0\right\}$

Let $\left(\pi_{y}, y \in \Omega\right)$ denote the steady-state distribution for the process $\left\{Y_{n}, n \geq\right.$ $0\}$ and $\Pi(z)$ denote its generating function, i.e., $\Pi(z) \equiv \sum_{j=0}^{\infty} \pi_{j} z^{j}$, where $\pi_{0}=$ $\pi_{0 I}+\pi_{0 B}$. From Ozawa $(1990), \Pi(z)$ is given by

$\Pi(z)=\frac{\frac{1}{2}\left\{1-\Theta_{0}(z)\right\} z^{K} \pi_{0}+\sum_{j=0}^{K-1}\left\{z^{K} \Theta_{j}(z)-z^{j} \Theta_{K}(z)\right\} \pi_{j}}{z^{K}-\Theta_{K}(z)}$,

where

$$
\begin{aligned}
\Theta_{0}(z) & =G_{0 I}^{*}\left(\lambda_{2}-\lambda_{2} z\right)=\frac{\lambda_{1}}{\lambda} G^{*}\left(\lambda_{2}-\lambda_{2} z\right)+\frac{\lambda_{2}}{\lambda} \Theta_{1}(z) \\
\Theta_{i}(z) & =G_{i}^{*}\left(\lambda_{2}-\lambda_{2} z\right) \\
& =\left\{H_{2}^{*}\left(\left(\lambda_{2}-\lambda_{2} z\right)+\lambda_{1}-\lambda_{1} G^{*}\left(\lambda_{2}-\lambda_{2} z\right)\right)\right\}^{i}, \quad i=1,2, \ldots, K
\end{aligned}
$$

There are $K$ unknown parameters $\left\{\pi_{j}, j=0,1,2, \ldots, K-1\right\}$ in formula (5). They can be obtained from the roots of the equation

$z^{K}-\Theta_{K}(z)=0,|z|<1$

and the following equation, which is derived from the normalizing condition for $\Pi(z)$ :

$K-\sum_{j=0}^{K-1}(K-j) \pi_{j}=\frac{\lambda_{2} \rho \pi_{0}}{2 \lambda(1-\rho)}$

From Lemmas 1 and 2 in Section 4 of Takács (1962), if $\rho<1$, then Equation (8) has exactly $K-1$ distinct roots in the region $|z|<1$.

Consider a regeneration interval, $\left(t_{n-1}, t_{n}\right]$. Let $N_{c y c l e}$ denote the number of Class 2 customers served during the interval in the case where $Y_{n-1} \neq 0 I$, and be equal to zero in the case where $Y_{n-1}=0 I$. Let $\xi_{K}^{(i)}$ denote the $i$ th factorial moment for $N_{c y c l e}$, and let $\xi_{K} \equiv \xi_{K}^{(1)} \cdot \xi_{K}$ and $\xi_{K}^{(2)}$ are given by

$$
\xi_{K}=\sum_{j=0}^{\infty} \min (j, K) \pi_{j}=K-\sum_{j=0}^{K-1}(K-j) \pi_{j}
$$




$$
\begin{aligned}
\xi_{K}^{(2)} & =\sum_{j=0}^{\infty} \min (j, K)\{\min (j, K)-1\} \pi_{j} \\
& =K(K-1)-\sum_{j=0}^{K-1}\{K(K-1)-j(j-1)\} \pi_{j}
\end{aligned}
$$

From (9) and (10), we obtain $\xi_{K}=\lambda_{2} \rho \pi_{0} /\{2 \lambda(1-\rho)\}$.

\subsection{Waiting time distributions and mean waiting times}

\section{(a) Waiting time distribution of Class 1 customers}

Let $L_{1}(z)$ denote the generating function of the distribution of the number of Class 1 customers in the system just after their departures, and let $W_{1}^{*}(s)$ denote the LST of the waiting time distribution of Class 1 customers. In terms of $L_{1}(z), W_{1}^{*}(s)$ is represented as $W_{1}^{*}(s)=L_{1}\left(1-s / \lambda_{1}\right) / H_{1}^{*}(s)$. Therefore, we derive $L_{1}(z)$ by using the results of the previous subsection.

Assume a steady-state process, and consider an interval $\left(t_{n}, t_{n+1}\right]$ between successive regeneration points. We introduce the following notations.

- $J_{n}^{(2)}$ : The number of Class 2 customers transferred at $t_{n} ; J_{n}^{(2)}=\min \left(Y_{n}, K\right)$. - $J_{n}^{(1)}$ : The number of Class 1 customers departed during interval $\left(t_{n}, t_{n+1}\right]$. - $t_{n, J_{n}^{(2)}}^{(2)}$ : Time point when the server completes all services for $J_{n}^{(2)}$ Class 2 customers transferred at $t_{n}$.

- $t_{n, j}^{(1)}, j=1, \ldots, J_{n}^{(1)}$ : The $j$ th departure time of Class 1 customers during interval $\left(t_{n}, t_{n+1}\right]$, where $t_{n, J_{n}^{(1)}}^{(1)}=t_{n+1}$, and $t_{n, 0}^{(1)} \equiv t_{n, J_{n}^{(2)}}^{(2)}$.

- $v_{n, j}^{(1)}, j=1, \ldots, J_{n}^{(1)}$ : The number of Class 1 customers who arrived during interval $\left(t_{n, j-1}^{(1)}, t_{n, j}^{(1)}\right]$.

- $X_{n, j}^{(1)}, j=0,1, \ldots, J_{n}^{(1)}$ : The number of Class 1 customers in the system just after time $t_{n, j}^{(1)}$.

$L_{1}(z)$ is represented as

$$
\begin{gathered}
\quad \frac{1}{C} L_{1}(z) \\
=\sum_{m=0}^{\infty} z^{m}\left[\sum_{j=1}^{K-1} \pi_{j} \sum_{k=1}^{\infty} \operatorname{Pr}\left(X_{n, 0}^{(1)}=k \mid J_{n}^{(2)}=j\right)\right. \\
\cdot \sum_{i=1}^{\infty} \operatorname{Pr}\left(X_{n, i}^{(1)}=m \mid X_{n, 0}^{(1)}=k\right)
\end{gathered}
$$




$$
\begin{aligned}
& +\sum_{j=K}^{\infty} \pi_{j} \sum_{k=1}^{\infty} \operatorname{Pr}\left(X_{n, 0}^{(1)}=k \mid J_{n}^{(2)}=K\right) \cdot \sum_{i=1}^{\infty} \operatorname{Pr}\left(X_{n, i}^{(1)}=m \mid X_{n, 0}^{(1)}=k\right) \\
& +\frac{\lambda_{1} \pi_{0 I}}{\lambda} \sum_{i=1}^{\infty} \operatorname{Pr}\left(X_{n, i}^{(1)}=m \mid X_{n, 0}^{(1)}=1\right) \\
& \left.+\frac{\lambda_{2} \pi_{0 I}}{\lambda} \sum_{k=1}^{\infty} \operatorname{Pr}\left(X_{n, 0}^{(1)}=k \mid Y_{n}=1\right) \cdot \sum_{i=1}^{\infty} \operatorname{Pr}\left(X_{n, i}^{(1)}=m \mid X_{n, 0}^{(1)}=k\right)\right]
\end{aligned}
$$

where $C$ is the normalizing constant. In the brackets on the right-hand side of the equation: the first term represents the probability that the number of Class 1 customers in the system just after their departure is $m$ in the case where $1 \leq Y_{n} \leq K-1$ and $J_{n}^{(2)}=Y_{n}$; the second term represents that probability in the case where $Y_{n} \geq K$ and $J_{n}^{(2)}=K$; the third term represents that probability in the case where $Y_{n}=0 I$ and a Class 1 customer arrived at $t_{n}$; and the fourth term represents that probability in the case where $Y_{n}=0 I$ and a Class 2 customer arrived at $t_{n}$.

Let the distribution function of $v_{n, j}^{(1)}$ be $v_{1}(i) \equiv \operatorname{Pr}\left(v_{n, j}^{(1)}=i\right) . X_{n, j}^{(1)}$ satisfies $X_{n, j}^{(1)}=X_{n, j-1}^{(1)}-1+v_{n, j}^{(1)}, j=1,2, \ldots, J_{n}^{(1)}$, therefore $\left\{X_{n, j}^{(1)}, 0 \leq j \leq J_{n}^{(1)}\right\}$ is a Markov chain having an absorbing state $0 ; J_{n}^{(1)}$ is the number of steps until the Markov chain reaches the absorbing state, hence $X_{n, J_{n}^{(1)}}^{(1)}=0$. From these points the following recursive expression for $\operatorname{Pr}\left(X_{n, j}^{(1)}=m \mid X_{n, 0}^{(1)}=k\right)$ is obtained.

$$
\begin{aligned}
& \operatorname{Pr}\left(X_{n, j}^{(1)}=m \mid X_{n, 0}^{(1)}=k\right) \\
& =\left\{\begin{array}{l}
0 \quad \text { for } j \leq k \text { and } m<k-j, \\
\sum_{r=k-j+1}^{m+1} v_{1}(m-r+1) \operatorname{Pr}\left(X_{n, j-1}^{(1)}=r \mid X_{n, 0}^{(1)}=k\right) \\
\text { for } j \leq k \text { and } m \geq k-j, \\
\sum_{r=1}^{m+1} v_{1}(m-r+1) \operatorname{Pr}\left(X_{n, j-1}^{(1)}=r \mid X_{n, 0}^{(1)}=k\right) \\
\text { for } j>k .
\end{array}\right.
\end{aligned}
$$

Using Equation (13), the following expression of $L_{1}(z)$ is obtained:

$$
\begin{aligned}
& \frac{1}{C} L_{1}(z) \\
= & \frac{H_{1}^{*}\left(\lambda_{1}-\lambda_{1} z\right)}{z-H_{1}^{*}\left(\lambda_{1}-\lambda_{1} z\right)}\left[\sum_{j=0}^{K-1}\left\{\left(H_{2}^{*}\left(\lambda_{1}-\lambda_{1} z\right)\right)^{j}-\left(H_{2}^{*}\left(\lambda_{1}-\lambda_{1} z\right)\right)^{K}\right\} \pi_{j}\right. \\
& \left.+\left(H_{2}^{*}\left(\lambda_{1}-\lambda_{1} z\right)\right)^{K}+\frac{\lambda_{1} \pi_{0 I}}{\lambda} z+\frac{\lambda_{2} \pi_{0 I}}{\lambda} H_{2}^{*}\left(\lambda_{1}-\lambda_{1} z\right)-\left(1+\pi_{0 I}\right)\right] .
\end{aligned}
$$


The value of the normalizing constant $C$ is obtained from the normalizing condition for $L_{1}(z)$ as follows:

$$
C=\frac{\lambda(1-\rho)}{\lambda_{1} \pi_{0 I}}
$$

From Equations (14) and (15), $W_{1}^{*}(s)$ is given by

$$
\begin{aligned}
W_{1}^{*}(s)= & \frac{\lambda(1-\rho) / \pi_{0 I}}{s-\lambda_{1}+\lambda_{1} H_{1}^{*}(s)}\left[1+\pi_{0 I}-\left(H_{2}^{*}(s)\right)^{K}\right. \\
& \left.-\sum_{j=0}^{K-1}\left\{\left(H_{2}^{*}(s)\right)^{j}-\left(H_{2}^{*}(s)\right)^{K}\right\} \pi_{j}-\left(\pi_{0 I} / \lambda\right)\left\{\lambda_{1}-s+\lambda_{2} H_{2}^{*}(s)\right\}\right] .
\end{aligned}
$$

\section{(b) Waiting time distribution of Class 2 customers}

Let $L_{2}(z)$ denote the generating function of the distribution of the number of Class 2 customers in the system immediately after their departure. The LST, $W_{2}^{*}(s)$, of the waiting time distribution of Class 2 customers is also obtained by using $L_{2}(z)$ as $W_{2}^{*}(s)=L_{2}\left(1-s / \lambda_{2}\right) / H_{2}^{*}(s)$.

Assume a steady-state process, and consider an interval $\left(t_{n}, t_{n+1}\right]$ between successive regeneration points. We introduce the following notations.

- $J_{n}^{(2)}$ : The number of Class 2 customers transferred at $t_{n} ; J_{n}^{(2)}=\min \left(Y_{n}, K\right)$. - $t_{n, j}^{(2)}, j=1, \ldots, J_{n}^{(2)}$ : The $j$ th departure time of Class 2 customers during interval $\left(t_{n}, t_{n+1}\right]$, and $t_{n, 0}^{(2)} \equiv t_{n}$.

- $v_{n, j}^{(2)}, j=1, \ldots, J_{n}^{(2)}$ : The number of Class 2 customers who arrived during interval $\left(t_{n, j-1}^{(2)}, t_{n, j}^{(2)}\right]$.

- $X_{n, j}^{(2)}, j=0,1, \ldots, J_{n}^{(2)}$ : The number of Class 2 customers in the system just after time $t_{n, j}^{(2)}$, where $X_{n, 0}^{(2)}=Y_{n}$.

Let the distribution of $v_{n, j}^{(2)}$ be $v_{2}(i) \equiv \operatorname{Pr}\left(v_{n, j}^{(2)}=i\right)$. Using these notations, $L_{2}(z)$ is represented as

$$
\begin{aligned}
& \frac{1}{C} L_{2}(z)=\sum_{m=0}^{\infty} z^{m} {\left[\frac{\lambda_{2} \pi_{0 I}}{\lambda} v_{2}(m)+\sum_{k=1}^{K-1} \pi_{k} \sum_{j=1}^{k} \operatorname{Pr}\left(X_{n, j}^{(2)}=m \mid X_{n, 0}^{(2)}=k\right)\right.} \\
&\left.+\sum_{k=K}^{\infty} \pi_{k} \sum_{j=1}^{K} \operatorname{Pr}\left(X_{n, j}^{(2)}=m \mid X_{n, 0}^{(2)}=k\right)\right]
\end{aligned}
$$


where $C$ is a normalizing constant. In the brackets on the right hand side of Equation (17): the first term represents the probability that the number of Class 2 customers in the system just after their departure is $m$ in the case where $Y_{n}=0 I$ and a Class 2 customer arrived at $t_{n}$; the second term represents that probability in the case where $1 \leq Y_{n} \leq K-1$; the third term represents that probability in the case where $Y_{n} \geq K$. Using the same procedures as those used for deriving $W_{1}^{*}(s), W_{2}^{*}(s)$ is given by

$$
\begin{aligned}
W_{2}^{*}(s)= & (1-\rho)+\frac{\lambda(1-\rho) / \pi_{0 I}}{\left(1-s / \lambda_{2}\right)^{K}\left\{s-\lambda_{2}+\lambda_{2} H_{2}^{*}(s)\right\}} \\
& \cdot\left[\left\{\left(H_{2}^{*}(s)\right)^{K}-\left(1-s / \lambda_{2}\right)^{K}\right\} \Pi\left(1-s / \lambda_{2}\right)\right. \\
& \left.-\sum_{k=0}^{K-1}\left\{\left(1-s / \lambda_{2}\right)^{k}\left(H_{2}^{*}(s)\right)^{K}-\left(1-s / \lambda_{2}\right)^{K}\left(H_{2}^{*}(s)\right)^{k}\right\} \pi_{k}\right] .
\end{aligned}
$$

\section{(c) Mean waiting times}

The mean waiting time $w_{1}$ is obtained by differentiating Equations (16) and (18) with respect to $s$ and putting $s \rightarrow 0+$, as follows:

$$
\begin{aligned}
w_{1} & =\frac{w_{0}}{1-\rho_{1}}-\frac{\lambda \rho_{2}(1-\rho) h_{2}}{\lambda_{2}\left(1-\rho_{1}\right) \pi_{0}} \xi_{K}^{(2)} \\
w_{2} & =\frac{w_{0}}{\left(1-\rho_{1}\right)(1-\rho)}+\frac{\lambda \rho_{1}(1-\rho) h_{2}}{\lambda_{2}\left(1-\rho_{1}\right) \pi_{0}} \xi_{K}^{(2)}
\end{aligned}
$$

where $\xi_{K}^{(2)}$ is given by (11). These results are coincident with the results of Ozawa (1990).

\section{A NON-ZERO WALKING TIME MODEL}

In this section, the LSTs of the waiting time distributions and the mean waiting times are derived for the two-queue model with non-zero walking times. In this model the number of customers in $\mathrm{Q}_{1}$ has a steady-state probability if and only if $\rho_{1}<1$; and that of customers in $\mathrm{Q}_{2}$ has a steady-state probability if and only if $\rho+\frac{\lambda_{2} u}{K}<1$. Hence, if $\rho_{1}<1$ and $\rho+\frac{\lambda_{2} u}{K} \geq 1$, the LST of the waiting time distribution of Class 1 customers and the mean waiting time can be derived by analyzing an $M / G / 1$ vacation model. In this section we assume that $\rho<1-\frac{\lambda_{2} u}{K}$ so that the numbers of customers in both the queues have steady-state probabilities.

An outline of the analysis is as follows. Considering the time points when the server departs from $Q_{1}$, the number of Class 2 customers in $Q_{2}$ forms a one-dimensional semi-Markov process. Using the steady-state distribution of the embedded Markov chain of this semi-Markov process, we can obtain the generating function of the distribution of the number of customers in 


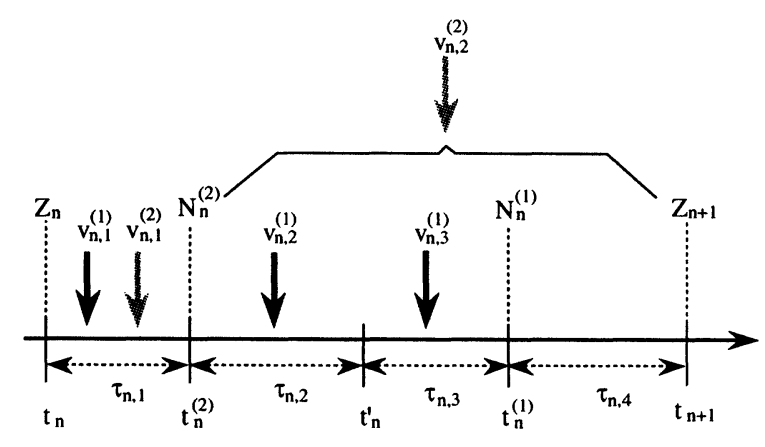

Figure 3 Behavior of the server during $\left(t_{n}, t_{n+1}\right]$.

each queue just before the time points when the server visits this queue. The LSTs of the waiting time distributions can be represented in terms of these generating functions.

\subsection{Embedded Markov chain}

Let $\left\{t_{n}\right\}$ be time points when the server departs from $\mathrm{Q}_{1}$ and $Z_{n}$ be the number of customers in $Q_{2}$ just after $t_{n}$. Since $Q_{1}$ is served according to an exhaustive service, the number of customers in $Q_{1}$ just after $t_{n}$ is always zero. Therefore, assuming the Poisson arrival processes and i.i.d. service time processes, the joint distribution of time length $\left(t_{n+1}-t_{n}\right)$ and $Z_{n+1}$ only depends on $Z_{n}$; letting $Z(t)$ be defined as $Z(t) \equiv \sum_{n=0}^{\infty} Z_{n} 1\left(t \in\left[t_{n}, t_{n+1}\right)\right)$, where $1(\cdot)$ is an indicator function, $\{Z(t)\}$ is a semi-Markov process, and $\left\{Z_{n}\right\}$ is its embedded Markov chain. Here, we consider only $\left\{Z_{n}\right\}$, and introduce the following notations (see Figure 3):

- $t_{n}^{(i)}$ : The time point when the server arrived at $\mathrm{Q}_{i}$ after $t_{n}, i=1,2$.

- $N_{n}^{(i)}$ : The number of customers in $\mathrm{Q}_{i}$ just before $t_{n}^{(i)}, i=1,2$.

- $v_{n, 1}^{(i)}$ : The number of Class $i$ customers arriving during $\left(t_{n}, t_{n}^{(2)}\right], i=1,2$.

- $t_{n}^{\prime}$ : The time point when the server departed from $\mathrm{Q}_{2}$ after $t_{n}$.

- $v_{n, 2}^{(1)}$ : The number of Class 1 customers arriving during $\left(t_{n}^{(2)}, t_{n}^{\prime}\right]$.

- $v_{n, 3}^{(1)}$ : The number of Class 1 customers arriving during $\left(t_{n}^{\prime}, t_{n}^{(1)}\right]$.

- $v_{n, 2}^{(2)}$ : The number of Class 2 customers arriving during $\left(t_{n}^{(2)}, t_{n+1}\right]$.

- $\tau_{n, 1}=t_{n}^{(2)}-t_{n}$ : Server's walking time from $\mathrm{Q}_{1}$ to $\mathrm{Q}_{2}$.

- $\tau_{n, 2}=t_{n}^{\prime}-t_{n}^{(2)}$ : Server's sojourn time at $\mathrm{Q}_{2}$.

- $\tau_{n, 3}=t_{n}^{(1)}-t_{n}^{\prime}$ : Server's walking time from $\mathrm{Q}_{2}$ to $\mathrm{Q}_{1}$.

- $\tau_{n, 4}=t_{n+1}-t_{n}^{(1)}$ : Server's sojourn time at $\mathrm{Q}_{1}$. 
Let the transition probability matrix of the embedded Markov chain $\left\{Z_{n}, n \geq\right.$ $0\}$ be denoted by $P=\left(p_{i, j}\right)$, where $p_{i, j} \equiv \operatorname{Pr}\left(Z_{n}=j \mid Z_{n-1}=i\right) .\left\{Z_{n}, n \geq 0\right\}$ satisfies the recursive formula $Z_{n+1}=\left[Z_{n}+v_{n, 1}^{(2)}-K\right]^{+}+v_{n, 2}^{(2)}$, and $\left(p_{i j}\right)$ satisfies the relation $p_{K+i, j}=p_{K, j-i}$. Hence $P$ is given by

$$
P=\begin{gathered}
0 \\
1 \\
\cdot \\
K+1 \\
\cdot
\end{gathered}\left(\begin{array}{cccc}
p_{0,0} & p_{0,1} & p_{0,2} & \cdot \\
p_{1,0} & p_{1,1} & p_{1,2} & \cdot \\
\cdot & \cdot & \cdot & \cdot \\
p_{K, 0} & p_{K, 1} & p_{K, 2} & \cdot \\
0 & p_{K, 0} & p_{K, 1} & \cdot \\
\cdot & \cdot & \cdot & \cdot
\end{array}\right)
$$

$\left(\tau_{n, 2}+\tau_{n, 3}+\tau_{n, 4}\right)$ is a delay cycle (Kleinrock 1976) for Class 1 customers: its initial delay is $\left(\tau_{n, 2}+\tau_{n, 3}\right)$, where $\tau_{n, 2}$ is the sum of service times of $\min \left(Z_{n}+v_{n, 1}^{(2)}, K\right)$ Class 2 customers and $\tau_{n, 3}$ is a walking time of the server. Therefore, for $i \leq K$, the generating function of $\left(p_{i, j}\right)$ with respect to $j$, denoted by $\Theta_{i}(z)$, is given by

$$
\begin{aligned}
& \Theta_{i}(z) \\
\equiv & \sum_{j=0}^{\infty} z^{j} p_{i, j} \\
= & U_{1}^{*}(g(z)) U_{2}^{*}(g(z))\left(H_{2}^{*}(g(z))\right)^{K} z^{-K+i}+U_{2}^{*}(g(z)) \\
& \cdot \sum_{\ell=0}^{K-i-1}\left\{\left(H_{2}^{*}(g(z))\right)^{i+\ell}-z^{-K+i+\ell}\left(H_{2}^{*}(g(z))\right)^{K}\right\} \varphi_{\ell}\left(G^{*}\left(\lambda_{2}-\lambda_{2} z\right)\right),(22
\end{aligned}
$$

where

$$
\begin{aligned}
g(z) & \equiv \lambda_{2}-\lambda_{2} z+\lambda_{1}-\lambda_{1} G^{*}\left(\lambda_{2}-\lambda_{2} z\right) \\
\varphi_{\ell}(z) & \equiv \sum_{k=0}^{\infty} z^{k} \operatorname{Pr}\left(v_{n, 1}^{(1)}=k, v_{n, 1}^{(2)}=\ell\right)=\frac{\left(-\lambda_{2}\right)^{\ell}}{\ell !} U_{1}^{*(\ell)}\left(\lambda_{1}-\lambda_{1} z+\lambda_{2}\right)
\end{aligned}
$$

and $G^{*}(s)$ is the LST of the busy period distribution for Class 1 customers, given by Equation (4).

Let $\left(\pi_{i}\right)$ denote the steady-state distribution for the process $\left\{Z_{n}, n \geq 0\right\}$, $\pi \equiv\left(\pi_{0} \pi_{1} \pi_{2} \ldots\right)$ denote its row vector, and $\Pi(z)$ denote its generating function. From the equilibrium equation $\pi P=\pi, \Pi(z)$ is given by

$$
\Pi(z) \equiv \sum_{i=0}^{\infty} z^{i} \pi_{i}=\frac{\sum_{j=0}^{K-1}\left\{z^{K} \Theta_{j}(z)-z^{j} \Theta_{K}(z)\right\} \pi_{j}}{z^{K}-\Theta_{K}(z)}
$$


where $\Theta_{i}(z)$ is given by Equation (22). $K$ unknown parameters $\left\{\pi_{j}, j=\right.$ $0,1, \ldots, K-1\}$ in Equation (25) can be obtained from the roots of the equation

$z^{K}-U_{1}^{*}(g(z)) U_{2}^{*}(g(z))\left(H_{2}^{*}(g(z))\right)^{K}=0, \quad|z|<1$,

and the following equation derived from the normalizing condition for $\Pi(z)$ :

$K-\sum_{j=0}^{K-1} \sum_{\ell=0}^{K-j-1}(K-j-\ell) \varphi_{\ell}(1) \pi_{j}=\frac{\lambda_{2} u}{1-\rho}$.

From Lemmas 1 and 2 in Section 4 of Takács (1962), if $\rho+\frac{\lambda_{2} u}{K}<1$, then Equation (26) has exactly $(K-1)$ distinct roots in the region $|z|<1$. The procedure descrived in the appendix of Ozawa (1990) can be applied to this case for calculating the values of the unknown parameters.

\subsection{Distributions of $N_{n}^{(1)}$ and $N_{n}^{(2)}$}

Let $F_{i}(z)$ denote the generating function of the distribution of the number of customers in $\mathrm{Q}_{i}$ just before the time points when the server visits the queue. In the following analysis, a steady-state process is assumed.

Since $v_{n, 3}^{(1)}$ is the number of Class 1 customers arriving during a walking time of the server from $\mathrm{Q}_{2}$ to $\mathrm{Q}_{1}$, it is independent from $v_{n, 1}^{(1)}, v_{n, 2}^{(1)}$, and $Z_{n}$. Therefore, from the equation $N_{n}^{(1)}=v_{n, 1}^{(1)}+v_{n, 2}^{(1)}+v_{n, 3}^{(1)}, F_{1}(z)$ is given by

$$
\begin{aligned}
F_{1}(z) \equiv & \sum_{i=0}^{\infty} z^{i} \operatorname{Pr}\left(N_{n}^{(1)}=i\right) \\
= & U_{1}^{*}\left(\lambda_{1}-\lambda_{1} z\right) U_{2}^{*}\left(\lambda_{1}-\lambda_{1} z\right)\left(H_{2}^{*}\left(\lambda_{1}-\lambda_{1} z\right)\right)^{K} \\
& +\sum_{k=0}^{K-1} \sum_{\ell=0}^{k}\left\{\left(H_{2}^{*}\left(\lambda_{1}-\lambda_{1} z\right)\right)^{k}-\left(H_{2}^{*}\left(\lambda_{1}-\lambda_{1} z\right)\right)^{K}\right\} \varphi_{\ell}(z) \pi_{k-\ell} .
\end{aligned}
$$

Since $N_{n}^{(2)}$ is equal to $\left(Z_{n}+v_{n, 1}^{(2)}\right)$ and $v_{n, 1}^{(2)}$ is independent of $Z_{n}, F_{2}(z)$ is given by the following formula.

$$
F_{2}(z) \equiv \sum_{i=0}^{\infty} z^{i} \operatorname{Pr}\left(N_{n}^{(2)}=i\right)=U_{1}^{*}\left(\lambda_{2}-\lambda_{2} z\right) \Pi(z),
$$

For use in the next subsection, we introduce some notations: let $N_{c y c l e}$ denote the number of Class 2 customers served during a cycle, which is a time 
period between consecutive visits of the server to $\mathrm{Q}_{1}$. Let $\xi_{K}^{(i)}$ denote the $i$ th factorial moment for $N_{\text {cycle }}$, and let $\xi_{K} \equiv \xi_{K}^{(1)}$. Since $\operatorname{Pr}\left(N_{n}^{(2)}=j\right)$ is given by

$\operatorname{Pr}\left(N_{n}^{(2)}=j\right)=\sum_{\ell=0}^{j} \operatorname{Pr}\left(Z_{n}=j-\ell, v_{n, 1}^{(2)}=\ell\right)=\sum_{\ell=0}^{j} \varphi_{\ell}(1) \pi_{j-\ell}$

$\xi_{K}$ and $\xi_{K}^{(2)}$ is obtained as follows:

$$
\begin{aligned}
\xi_{K} & =\sum_{j=0}^{\infty} \min (j, K) \operatorname{Pr}\left(N_{n}^{(2)}=j\right)=K-\sum_{j=0}^{K-1} \sum_{\ell=0}^{j}(K-j) \varphi_{\ell}(1) \pi_{j-\ell} \\
\xi_{K}^{(2)} & =\sum_{j=0}^{\infty} \min (j, K)\{\min (j, K)-1\} \operatorname{Pr}\left(N_{n}^{(2)}=j\right) \\
& =K(K-1)-\sum_{j=0}^{K-1} \sum_{\ell=0}^{j}\{K(K-1)-j(j-1)\} \varphi_{\ell}(1) \pi_{j-\ell}
\end{aligned}
$$

From Equations (27) and (31), we obtain $\xi_{K}=\lambda_{2} u /(1-\rho)$.

Since the maximum number of Class 2 customers served during one cycle is $K, \xi_{K}$ must be less than $K$ if $\mathrm{Q}_{2}$ is not saturated. From this, we obtain the condition $\frac{\lambda_{2} u}{1-\rho}<K$, in which the number of customers in $\mathrm{Q}_{2}$ has a steady-state probability.

\subsection{Waiting time distributions and mean waiting times}

(a) Relation between the waiting time distribution and the distribution of the number of customers in a queue

Consider a polling model with Poisson arrival processes and i.i.d. service time processes. Let $X_{0}^{(i)}$ denote the number of customers in queue $i$ just before a time point when the server visited this queue. The $\operatorname{LST}, W_{i}^{*}(s)$, of the waiting time distribution of Class $i$ customers is represented in terms of $F_{i}(z)$, which is the generating function of the distribution of $X_{0}^{(i)}$, as follows:

- If queue $i$ is served according to an exhaustive service,

$$
W_{i}^{*}(s)=\frac{1-\rho_{i}}{F_{i}^{\prime}(1)} \cdot \frac{1-F_{i}\left(1-s / \lambda_{i}\right)}{H_{i}^{*}(s)-1+s / \lambda_{i}}
$$


- If queue $i$ is served according to a gated-type $K$-limited service,

$$
\begin{aligned}
W_{i}^{*}(s)= & \frac{\lambda_{2} C_{K L}}{\left(1-s / \lambda_{i}\right)^{K}\left\{s-\lambda_{i}+\lambda_{i} H_{i}^{*}(s)\right\}} \\
& \cdot\left[\left\{\left(H_{i}^{*}(s)\right)^{K}-\left(1-s / \lambda_{i}\right)^{K}\right\} F_{i}\left(1-s / \lambda_{i}\right)\right. \\
& \left.-\sum_{k=0}^{K-1}\left\{\left(1-s / \lambda_{i}\right)^{k}\left(H_{i}^{*}(s)\right)^{K}-\left(1-s / \lambda_{i}\right)^{K}\left(H_{i}^{*}(s)\right)^{k}\right\} x_{i}(k)\right]
\end{aligned}
$$

In these formulas, $\lambda_{i}$ and $H_{i}^{*}(s)$ are the arrival intensity and the LST of the service time distribution of Class $i$ customers; $C_{K L} \equiv\left\{K-\sum_{k=0}^{K-1}(K-\right.$ $\left.k) x_{i}(k)\right\}^{-1}$ and $x_{i}(k) \equiv \operatorname{Pr}\left(X_{0}^{(i)}=k\right)$. Note that, under the condition where $F_{i}(z)$ is given, $W_{i}^{*}(s)$ depends only on the service discipline of queue $i$. Equation (33) is given by Watson (1984). Equation (34) is derived as follows. Consider a cycle, and let $X_{j}^{(i)}$ be the number of customers in queue $i$ just after the time when the $j$ th customer departed from this queue during the cycle. The generating function, $L_{i}(z)$, of the distribution of the number of customers in queue $i$ just after their departures is given by

$$
\frac{1}{C} L_{i}(z)=\sum_{m=0}^{\infty} z^{m} \sum_{k=1}^{\infty} \sum_{j=1}^{\min (k, K)} \operatorname{Pr}\left(X_{j}^{(i)}=m \mid X_{0}^{(i)}=k\right) \operatorname{Pr}\left(X_{0}^{(i)}=k\right)
$$

where $C$ is the normalizing constant. $\operatorname{Pr}\left(X_{j}^{(i)}=m \mid X_{0}^{(i)}=k\right)$ satisfies the following recursive formula:

$$
\begin{aligned}
& \operatorname{Pr}\left(X_{j}^{(i)}=m \mid X_{0}^{(i)}=k\right) \\
= & \begin{cases}0, & m<k-j, \\
\sum_{r=k-j+1}^{m+1} v(m-r+1) \operatorname{Pr}\left(X_{j-1}^{(i)}=r \mid X_{0}^{(i)}=k\right), & m \geq k-j,\end{cases}
\end{aligned}
$$

where $v(\ell) \equiv \frac{\lambda_{i}^{\ell}}{\ell !} e^{-\lambda_{i}}$. Hence $L_{i}(z)$ is given by

$$
\begin{aligned}
\frac{1}{C} L_{i}(z)= & \frac{H_{i}^{*}\left(\lambda_{i}-\lambda_{i} z\right)}{z^{K}\left\{z-H_{i}^{*}\left(\lambda_{i}-\lambda_{i} z\right)\right\}}\left[\left\{z^{K}-\left(H_{i}^{*}\left(\lambda_{i}-\lambda_{i} z\right)\right)^{K}\right\} F_{i}(z)\right. \\
& \left.-\sum_{k=0}^{K}\left\{z^{K}\left(H_{i}^{*}\left(\lambda_{i}-\lambda_{i} z\right)\right)^{k}-z^{k}\left(H_{i}^{*}\left(\lambda_{i}-\lambda_{i} z\right)\right)^{K}\right\} x_{i}(k)\right]
\end{aligned}
$$


From the normalizing condition for $L_{i}(z), C$ is given by

$C=\left\{K-\sum_{k=0}^{K-1}(K-k) x_{i}(k)\right\}^{-1}=C_{K L}$

Substituting (37) into $W_{i}^{*}(s)=L_{i}\left(1-s / \lambda_{i}\right) / H_{i}^{*}(s)$, formula (34) is obtained.

\section{(b) LSTs of the waiting time distributions}

Since the two-queue model is a polling model, the results of the previous subsection can be used to derive the LSTs of the waiting time distributions. In our model $C_{K L}$ in Equation (34) is equal to $\xi_{K}^{-1}=\left(\frac{\lambda_{2} u}{1-\rho}\right)^{-1}$, therefore we get the following formulas from Equations (28), (29), (33), and (34):

$$
\begin{aligned}
W_{1}^{*}(s)= & \frac{(1-\rho) / u}{s-\lambda_{1}+\lambda_{1} H_{1}^{*}(s)}\left[1-U_{1}^{*}(s) U_{2}^{*}(s)\left(H_{2}^{*}(s)\right)^{K}\right. \\
& \left.-\sum_{k=0}^{K-1} \sum_{\ell=0}^{k}\left\{\left(H_{2}^{*}(s)\right)^{k}-\left(H_{2}^{*}(s)\right)^{K}\right\} \varphi_{\ell}\left(1-s / \lambda_{1}\right) \pi_{k-\ell}\right], \\
W_{2}^{*}(s)= & \frac{(1-\rho) / u}{\left(1-s / \lambda_{2}\right)^{K}\left\{s-\lambda_{2}+\lambda_{2} H_{2}^{*}(s)\right\}} \\
& \cdot\left[\left\{\left(H_{2}^{*}(s)\right)^{K}-\left(1-\frac{s}{\lambda_{2}}\right)^{K}\right\} U_{1}^{*}(s) \Pi\left(1-\frac{s}{\lambda_{2}}\right)\right. \\
& -\sum_{k=0}^{K-1} \sum_{\ell=0}^{k}\left\{\left(1-\frac{s}{\lambda_{2}}\right)^{k}\left(H_{2}^{*}(s)\right)^{K}\right. \\
& \left.\left.-\left(1-\frac{s}{\lambda_{2}}\right)^{K}\left(H_{2}^{*}(s)\right)^{k}\right\} \varphi_{\ell}(1) \pi_{k-\ell}\right] .
\end{aligned}
$$

\section{(c) Mean waiting times}

The mean waiting times are obtained by differentiating Equations (39) and (40) with respect to $s$ and putting $s \rightarrow 0$, as follows:

$$
\begin{aligned}
w_{1}= & \frac{w_{0}}{1-\rho_{1}}+\frac{\rho_{2} u_{2}}{1-\rho_{1}}+\frac{K h_{2}(1-\rho) u_{1}}{\left(1-\rho_{1}\right) u}+\frac{(1-\rho) u^{(2)}}{2\left(1-\rho_{1}\right) u} \\
& +\frac{(1-\rho) h_{2}^{(2)}}{2\left(1-\rho_{1}\right) u} \xi_{K}^{(2)}-\frac{(1-\rho) h_{2}}{\lambda_{2}\left(1-\rho_{1}\right) u} \zeta_{K}^{(2)} \\
w_{2}= & \frac{w_{0}}{\left(1-\rho_{1}\right) \alpha_{K}}+\frac{\rho_{2} u}{\left(1-\rho_{1}\right) \alpha_{K}}-\frac{K(1-\rho) \rho_{1} u_{1}}{\lambda_{2}\left(1-\rho_{1}\right) u}+\frac{(1-\rho) u^{(2)}}{2\left(1-\rho_{1}\right) \alpha_{K} u} \\
& -\frac{1-\rho}{2 \lambda_{2}^{2} u}\left\{\frac{(1-\rho)\left(1-\rho_{1}+\rho_{2}\right)}{\left(1-\rho_{1}\right) \alpha_{K}}-\left(1+\rho_{2}\right)\right\} \xi_{K}^{(2)}
\end{aligned}
$$




$$
+\frac{(1-\rho)^{2} \rho_{1}}{\lambda_{2}^{2}\left(1-\rho_{1}\right) \alpha_{K} u} \zeta_{K}^{(2)}
$$

where $\xi_{K}^{(2)}$ is given by Equation (32), and $\alpha_{K}$ and $\zeta_{K}^{(2)}$ are given by

$$
\begin{aligned}
\alpha_{K} & \equiv(1-\rho)-\frac{\lambda_{2} u}{K} \\
\zeta_{K}^{(2)} & \equiv \sum_{k=0}^{K-1} \sum_{\ell=0}^{k}(K-k)(\ell+1) \varphi_{\ell+1}(1) \pi_{k-\ell}
\end{aligned}
$$

$w_{1}$ and $w_{2}$ satisfy the following pseudo-conservation law (Everitt 1986, Everitt 1989):

$$
\begin{aligned}
& \rho_{1} w_{1}+\rho_{2}\left(1-\frac{\lambda_{2} u}{(1-\rho) K}\right) w_{2} \\
= & \frac{\rho w_{0}}{1-\rho}+\frac{\rho u^{(2)}}{2 u}+\frac{\rho_{2} \rho u}{1-\rho}-\frac{\rho_{2}\left(1+\rho_{2}\right)}{2} \cdot \frac{\xi_{K}^{(2)}}{\lambda_{2} K} .
\end{aligned}
$$

\section{NUMERICAL EXAMPLES}

Using numerical examples, we show how the mean waiting times vary with parameter $K$ for a case of non-zero walking times. Here it is assumed that both $H_{1}(t)$ and $H_{2}(t)$ are exponential distributions, whose mean values are one (i.e. $h_{1}=h_{2}=1$ ), and that both $U_{1}(t)$ and $U_{2}(t)$ are unit distributions, whose mean values are 0.1 (i.e. $u_{1}=u_{2}=0.1$ ). We further assume that $\lambda_{1}=\lambda_{2}$. The mean waiting times of Class 1 customers, $w_{1}$, and those of Class 2 customers, $w_{2}$, are plotted in Figure 4 . For each value of $\rho, w_{1}$ increases and $w_{2}$ decreases monotonically as $K$ increases.

\section{CONCLUSIONS}

A single-server model was analyzed using a delay cycle technique, and the LSTs of the waiting time distributions and the mean waiting times were obtained. The model had two queues: one is served according to an exhaustive service, and the other served according to a gated-type $K$-limited service. The results for a model with mixed exhaustive and 1-limited services were given as a special case where $K=1$. The results for a model with mixed exhaustive and gated services were given as a special case where $K=\infty$ (Ozawa 1988).

These results are useful for evaluating integrated services systems that handle various kinds of inputs. They can also be used to verify the accuracy of approximation formulas of polling models with mixed service disciplines. 


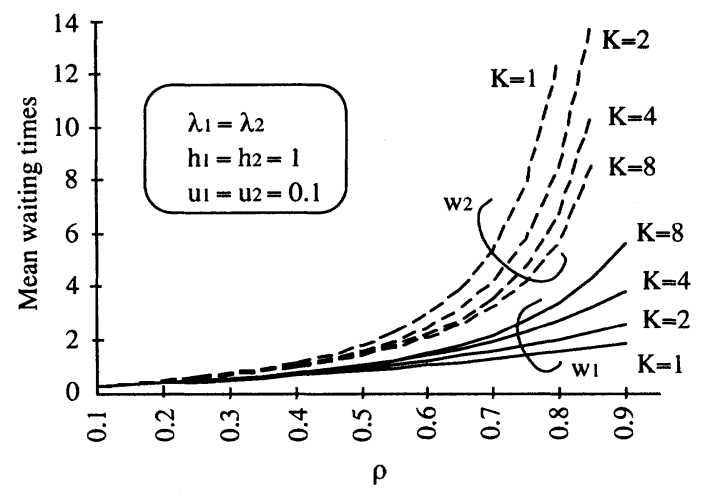

Figure 4 Mean waiting times for a case of non-zero walking times.

\section{Acknowledgments}

I would like to thank anonymous referees for their valuable comments.

\section{REFERENCES}

Avi-Itzhak, B., Maxwell, W. L., and Miller, L. W. (1965) Queueing with alternating priorities. Operations Research, 13 (2), 306-18.

Boxma, O. J. (1984) Two symmetric queues with alternating service and switching time. Performance '84 (ed. E. Gelenbe), Elsevier Science, Amsterdam , 409-31.

Boxma, O. J. and Groenendijk, W. P. (1987) Pseudo-conservation laws in cyclic-service systems. Journal of Applied Probability, 24 (4), 949-64.

Cohen, J. W. and Boxma, O. J. (1981) The M/G/1 queue with alternating service formulated as a Riemann-Hilbert problem. Performance ' 81 (ed. F. J. Kylstra), North-Holland, Amsterdam, 181-99.

Eisenberg, M. (1971) Two queues with changeover times. Operations Research, 19 (2), 386-401.

Eisenberg, M. (1979) Two queues with Alternating Service. SIAM Journal on Applied Mathematics, 36 (2), 287-303.

Everitt, D. (1986) A conservation-type law for the token ring with limited service. British Telecom Technology Journal, 4 (2), 51-61.

Everitt, D. (1989) A note on the pseudo-conservation laws for cyclic service systems with limited service disciplines. IEEE Transactions on Communications, 37 (7), 781-3.

Fuhrmann, S. W. and Wang, Y. T. (1988) Analysis of cyclic service systems with limited service: bounds and approximations, Performance Evaluation, 9, 35-54.

Groenendijk, W. P. (1988) Waiting-time approximations for cyclic-service sys- 
tems with mixed service strategies. ITC-12 (ed. M. Bonatti), Elsevier Science, Amsterdam, 1434-41.

Jaiswal, N. K. (1986) Priority Queues. Academic Press, New York.

Kleinrock, L. (1976) Queueing Systems Vol. II : Applications, John Wiley, New York.

Kuczura, A. (1973) Piecewise Markov processes, SIAM Journal on Applied Mathematics, 24 (2), 169-81.

Lee, D.-S. (1996) A two-queue model with exhaustive and limited service disciplines. Stochastic Models, 12 (2), 285-305.

Ozawa, T. (1988) Analysis of a single server model with two queues having different service disciplines. The Transactions of the IEICE, J71-A (5), 1193-1200 (in Japanese). (English translation version: Electronics and Communications in Japan (Part 3), 74 (3), 1990, 18-27.)

Ozawa, T. (1990) Alternating service queues with mixed exhaustive and $K$ limited services. Performance Evaluation, 11, 165-75.

Skinner, C. E. (1967) A priority queuing system with server-walking times. Operations Research, 15 (2), 278-85.

Takács, L. (1962) Introduction to the Theory of Queues, Oxford University Press, New York.

Takács, L. (1968) Two queues attended by a single server. Operations Research, 16 (3), 639-650.

Takagi, H. (1986) Analysis of Polling Systems. The MIT Press, Cambridge.

Takagi, H. (1988) Queuing analysis of polling models. ACM Computing Surveys, 20 (1), 5-28.

Takagi, H. (1989) Analysis of polling systems with a mixture of exhaustive and gated service discipline. Journal of the Operations Research Society of Japan, 32 (4), 450-61.

Watson, K. S. (1984) Performance evaluation of cyclic service strategies - a survey. Performance '84 (ed. E. Gelenbe), Elsevier Science, Amsterdam, 521-33.

\section{BIOGRAPHY}

Toshihsa Ozawa received the B.E. degree in information science, M.E. degree in system science, and Dr. degree in information science from Tokyo Institute of Technology in 1984, 1986, and 1996, respectively. In 1986 he joined NTT laboratories, where he is currently engaged in research of performance evaluation of multimedia networks. 